\title{
La posizione dell'italiano come lingua seconda nel sistema educativo sloveno e le proposte per la sua rivitalizzazione tra i giovani dell'area bilingue
}

\author{
Rok Kobal \\ Osnovna šola Koper \\ rok.kobal88@gmail.com
}

Položaj italijanščine kot drugega jezika v slovenskem šolskem sistemu in predlogi za njeno revitalizacijo med mladimi na dvojezičnem območju

Glavni namen prispevka je ponuditi bralcu predstavitev zaskrbljujočega stanja italijanščine kot drugega jezika yzdolž slovenske obale in predstaviti konkretne rešitve za revitalizacijo in izboljšanje italijanščine $\mathrm{v}$ šolah slovenske Istre. $V$ ta namen sta $\mathrm{v}$ članku predstavljena dva primera dobre prakse: valižanski in baskovski model, katerih uspeh nas lahko navdaja z upanjem, da tudi za italijanski jezik v slovenski Istri še vedno obstajajo možnosti za učinkovit poseg.

Ključne besede: italijanski jezik, slovenska Istra, jezikovna revitalizacija, valižanski model, baskovski model

The Position of Italian as a Second Language in the Slovenian School System and the Proposals for Its Revitalization among Young People in the Bilingual Area

The objective of this article is to offer the reader a presentation of the alarming position of Italian as a second language along the Slovenian coast and to propose a variety of possible solutions for its revitalization and improvement in schools in Slovenian Istria. For this purpose are presented two examples of good practice: the Welsh and Basque model. The success of these two models can fill us with hope that in the current situation, there is still room for an effective intervention for the Italian language.

Keywords: Italian language, Slovenian Istria, linguistic revitalization, Welsh model, Basque model

$\mathrm{N}$

ella società odierna, caratterizzata da una densa rete di interconnessioni in ambito globale, la conoscenza delle lingue seconde e straniere si mostra come una competenza sempre più importante e necessaria. In un periodo in cui i contatti fra le nazioni appaiono ogni volta più intensi e si realizzano a diversi livelli, come quello economico, politico e culturale, padroneggiare una lingua seconda e/o straniera, tanto nella sfera professionale quanto in quella personale, rappresenta un punto di partenza non solo consigliabile, ma ormai sempre più necessario. La conoscenza delle lingue occupa un ruolo centrale nella comunicazione internazionale e contribuisce ad abbattere i pregiudizi e le discriminazioni. In altre parole, la diversità linguistica è uno strumento per ottenere una migliore comprensione interculturale ed è un elemento chiave nel ricco patrimonio culturale del nostro continente. La politica europea promuove apertamente l'apprendimento e l'insegnamento delle lingue seconde/straniere con l'obiettivo di assicurare e di sostenere il plurilinguismo dei cittadini comunitari. Per questo motivo, la padronanza delle diverse lingue rappresenta una base fondamentale nella costruzione dello spazio comune europeo, della cooperazione internazionale e della mobilità transfrontaliera (Jagodic 2013, 40). 
L'obiettivo principale del presente articolo è di offrire al lettore una presentazione breve della posizione dell'italiano come lingua seconda nel sistema educativo sloveno dell'area bilingue lungo la fascia costiera, di rilevare la situazione preoccupante di oggi e di proporre delle proposte concrete per la rivitalizzazione e il miglioramento della lingua italiana nelle scuole dell'Istria slovena che negli ultimi vent'anni si trova in crisi.

\section{Metodologia}

La seguente analisi sul tema della crisi dell'ita-

liano L2 nel territorio bilingue e delle buone pratiche effettuate nell'ambito della rivitalizzazione delle lingue minoritarie è stata condotta usando sia l'approccio quantitativo sia quello qualitativo. L'obiettivo della parte quantitativa era soprattutto quello di precisare il periodo in cui il livello di conoscenza dell' italiano è cominciato a calare. A tale scopo è stata condotta una breve analisi sulla prestazione degli studenti sloveni nel territorio bilingue all'esame di maturità di lingua italiana tra gli anni 1995 e 2018 che offre una valutazione obiettiva della padronanza linguistica. Anche se i candidati che accedono all'esame di maturità possono scegliere tra il livello base oppure quello avanzato, sono stati utilizzati, ai fini della nostra analisi, solamente i risultati ottenuti al livello avanzato poiché questo dovrebbe riflettere una competenza più elevata nella lingua italiana. Abbiamo deciso di indagare sul numero di candidati che hanno sostenuto l'esame tra gli anni 1995 e 2018 , sulla percentuale dei candidati che all'esame hanno conseguito 8 (il punteggio massimo) o 6 punti, sulla percentuale dei punti necessari per ottenere un voto positivo e sul voto medio ottenuto dagli studenti. Tutti i dati sono stati derivati dal sito Internet del Centro nazionale per gli esami (RIC).

Nella seconda parte della ricerca è stata presentata la grande differenza tra la posizione dell'italiano come lingua seconda e dell' inglese come lingua straniera nelle scuole dell'Istria slovena. A tale scopo sono stati presentati il numero delle lezioni destinate alla singola lingua e le principali differenze nel loro insegnamento. Le informazioni sono state tratte dal Libro bianco su istruzione e formazione / Bela knjiga o vzgoji in izobraževanju v Republiki Sloveniji (Krek e Metljak 20II) nonché dai curricoli scolastici per l'italiano L2 e l'inglese LS.

Nella seguente parte dell'analisi sono stati esaminati attentamente due buoni esempi di rivitalizzazione linguistica in Europa. Con l'aiuto delle ricerche svolte in Galles e nei Paesi Baschi, si è potuto eseguire così un'analisi comparativa tra il sistema gallese e quello basco, i due modelli che hanno ottenuto negli ultimi anni il maggiore successo nell'ambito della pianificazione e dello sviluppo delle lingue minoritarie. Sulla base delle constatazioni e dei risultati derivati dall'analisi, sono state offerte poi delle proposte concrete per una strategia d'intervento a favore della lingua italiana nell'Istria slovena.

\section{Lingua seconda ( $\left.\mathrm{L}_{2}\right)$}

Generalmente, la seconda lingua è definita una lingua che viene appresa dopo la lingua materna, nel paese in cui essa viene usata per la comunicazione. Si tratta di una lingua che può essere imparata dentro e/o fuori da un ambiente formale (scolastico), cioè una lingua che lo studente può trovare anche fuori dalla scuola, come nel caso di un italiano che studi il francese in Francia, un algerino che studi l'inglese in Inghilterra oppure uno sloveno che impara l'italiano frequentando le scuole del territorio bilingue dei comuni costieri in Slovenia dove l' italiano rappresenta la lingua dell'ambiente sociale (Luise 2006, 42). Secondo i glottodidatti, l'italiano è una lingua seconda quando viene insegnato a parlanti non italofoni sul territorio dove essa è utilizzata come lingua di comunicazione quotidiana - ad esempio, Italia, Canton Ticino, aree bilingui dell'Istria (Balboni 1994, I3). Come spiega Balboni $(2008,58)$, a differenza della lingua straniera, nel processo di acquisizione di una lingua seconda molto dell' input linguistico su cui si lavora deriva dall'esterno, cioè dal mondo extralinguistico, ed è spesso portato a scuola dagli stessi studenti.

In seguito, Balboni (2008, 200 e 2014, 19) rileva che dal punto di vista glottodidattico, 
nell' insegnamento della seconda lingua il discente è continuamente in contatto con la $\mathrm{L}_{2}$ e il docente non ha un controllo completo dell' input che viene fornito allo studente. Per questo motivo, l'insegnante non è consapevole di quanto e cosa il discente abbia appreso spontaneamente dall'ambiente nella vita quotidiana in cui egli è esposto alla lingua viva, non strutturata e graduata, non spiegata e commentata. Nonostante gli sforzi fatti dagli insegnanti per creare in classe situazioni reali, l'ambiente scolastico in cui l'alunno apprende la lingua resta perlopiù artificiale.

Per gli appartenenti al gruppo nazionale maggioritario l'apprendimento della lingua seconda è di solito successivo all'acquisizione della lingua materna, mentre per gli appartenenti al gruppo minoritario l'acquisizione delle due lingue spesso avviene simultaneamente grazie agli stimoli dall'ambiente maggioritario (Pišot e Čok 1990, 158).

\section{Italiano come $\mathrm{L}_{2}$}

Con la legge del 1959 l'italiano diventa materia obbligatoria nelle scuole primarie e secondarie nel territorio nazionalmente misto e bilingue lungo la costa slovena (Čok 2009, 89). Questo modello di educazione bilingue permette agli alunni che frequentano la scuola dell'obbligo e quella superiore dell'area plurietnica di seguire per due ore settimanali la lingua italiana come materia d'obbligo. Come spiegano Limon e Novak-Lukanovič $(2012,30)$ si tratta di un modello che aspira al mantenimento della lingua d'origine, con il doppio obiettivo di acquisire una lingua di comunicazione più efficace, ma allo stesso tempo mantenere la propria lingua. Grazie a questo modello che ambisce all'arricchimento del repertorio linguistico, i bambini monolingui possono raggiungere un bilinguismo funzionale in maniera più efficiente.

Gli allievi che frequentano le scuole con lingua d'insegnamento slovena nel territorio bilingue lungo la costa slovena apprendono l'italiano come lingua seconda ovvero come lingua dell'ambiente sociale. In queste zone i ragazzi appartenenti al gruppo maggioritario hanno la possibilità di stare continuamente in stretto contatto con gli italofoni della minoranza etnica e di usare la lingua italiana pure al di fuori degli istituti scolastici. Nelle scuole slovene gli alunni seguono le lezioni d'italiano come seconda lingua obbligatoria dal primo triennio della scuola dell'obbligo fino all'esame di maturità o di licenza alla fine della scuola media superiore (Zudič Antonič 2007, 16).

Secondo i parametri del Consiglio d'Europa alla fine della scuola elementare gli allievi dell'Istria slovena dovrebbero raggiungere il livello della conoscenza della lingua italiana $\mathrm{A}_{2}$ o BI, mentre il maturando alle superiori dovrebbe raggiungere almeno il livello $\mathrm{B}_{2}$ o $\mathrm{C}_{\mathrm{I}}$. Sfortunatamente, la situazione reale - come vedremo di seguito analizzando la situazione attuale dell' $i$ taliano L2 tra gli allievi nelle scuole slovene del territorio bilingue - spesso non coincide con gli obiettivi attesi.

\section{La crisi dell'italiano L2 nell'area bilingue}

Gli obiettivi menzionati sopra erano sicuramente raggiungibili e realizzabili fino a venti anni fa, quando il livello della conoscenza della lingua italiana era ancora soddisfacente. In quel periodo gli alunni e gli studenti frequentavano le lezioni d'italiano con gioia e piacere non avendo bisogno di corsi linguistici e di lezioni aggiuntive per soddisfare gli obiettivi del curricolo scolastico e per ottenere un voto positivo. I bambini amavano l'italiano usandolo ogni giorno, guardando programmazioni televisive italiane e imparando canzoni dei loro cantanti italiani preferiti. In questo modo i ragazzi entravano a scuola con una conoscenza dell' italiano piuttosto elevata grazie alla quale potevano facilmente seguire le lezioni di L2. Gli abitanti dell'Istria slovena erano allora costantemente in contatto con la cultura italiana conoscendo gli attori, gli scrittori, i poeti e i cantanti italiani, seguivano e imitavano lo stile di vita degli italiani, conoscevano le loro abitudini, il loro modo di pensare, il loro cibo e la loro politica. Per questo motivo non sorprendono i risultati dell'indagine svol- 
ta da Novak-Lukanovič (1998) che ha compreso 700 persone viventi nel territorio bilingue. Il $75,3 \%$ degli intervistati ha, indipendentemente dalla loro nazionalità, confermato che gli alunni sloveni dell'area bilingue avrebbero dovuto studiare l'italiano come lingua dell'ambiente sociale. Tra le ragioni di questo hanno accentuato soprattutto la vita nel territorio nazionalmente misto (il 23,5\%), la convivenza delle due nazioni (il 21,3\%), l'immediata vicinanza dell'Italia (il 20\%) e l'importanza delle lingue (il 19,3\%). Le affermazioni fatte in precedenza possono essere confermate pure dall' indagine svolta da Kompa-

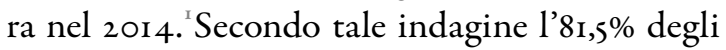
intervistati sopra i 25 anni ha, infatti, dichiarato di aver studiato l'italiano a scuola con piacere, mentre tra le persone alle quali l'italiano non rappresentava una materia piacevole, erano soprattutto i giovani sotto i 25 anni.

Sfortunatamente, negli ultimi vent'anni la posizione della lingua italiana tra i giovani dell'Istria slovena è cambiata drasticamente. Come spiegano Miklič e Ožbot (200I, II7), intorno al 2000 si sono levate le prime voci d'insoddisfazione, quando le insegnanti d'italiano nelle elementari hanno cominciato a notare un declino, nei giovani sloveni, nella conoscenza del lessico italiano. Gli insegnanti dell'italiano L2 che lavorano nelle scuole slovene dell'area bilingue concordano sul fatto che gli studenti bilingui capaci di esprimersi in entrambe le lingue in modo equilibrato oggi rappresentino una vera rarità. Secondo la loro opinione, il livello di conoscenza della lingua italiana è gradualmente calato così in basso che i giovani nati dopo il I990 non possono essere più identificati come individui bilingui.

Per precisare meglio il periodo in cui il livello di conoscenza dell' italiano è cominciato a calare, nel paragrafo seguente sono presentati i risultati di una breve analisi sulla prestazione degli studenti sloveni nel territorio bilingue all'esame di maturità di lingua italiana tra gli anni 1995 e 2018.

L'indagine intitolata Je slovenska Istra še dvojezična? (L'Istria slovena è ancora bilingue?) è stata svolta da Mojca Kompara nel 2014.

\section{Esame di maturità di lingua italiana}

In Slovenia, l'esame di maturità generale di lingua italiana è destinato ai candidati che hanno scelto l'italiano come materia d'esame facoltativa e offre una valutazione obiettiva della padronanza della lingua italiana in tutti i suoi usi rappresentando così un'indicazione precisa del periodo in cui si è cominciato ad assistere a un declino, nei giovani sloveni, nella conoscenza dell'italiano.

Ai fini della nostra analisi sono stati utilizzati i risultati ottenuti al livello avanzato poiché questo dovrebbe riflettere una competenza più elevata nella lingua italiana. Analizzando i dati nella tabella I, si possono notare alcuni punti molto interessanti che hanno contrassegnato il periodo tra il 1995, quando è stato eseguito il primo esame di maturità in Slovenia, e il 2018.

Dalla quinta colonna si può notare che dopo il 2008 il numero degli studenti che si sono presentati all'esame di maturità di lingua italiana a livello avanzato è cominciato a calare rapidamente raggiungendo i9 nel 2018 . In altre parole, tra gli anni 1995 e 2008 i candidati erano in media I04,2I, mentre tra gli anni 2009 e 2018 il numero medio si è fermato su 46,40 . Questi dati preoccupanti riflettono sicuramente una situazione allarmante della lingua italiana nell'Istria slovena che negli ultimi dieci anni ha subito un calo di popolarità tremendo. Analizzando i dati nella quarta colonna in cui è specificata la percentuale dei punti necessari per il voto positivo, si possono trarre conclusioni simili. Come si può notare, tra gli anni 1999 e 2006 , la soglia media per il voto positivo era del 55,62\% (nel 1999 ha raggiunto persino il 60\%), mentre tra gli anni 2007 e 2018 è rimasta sempre la stessa raggiungendo il 50\%. Poiché la soglia minima per il voto positivo viene stabilita in base alla prestazione degli studenti nel singolo anno (migliori sono i risultati, più alta è la soglia minima), si può affermare con certezza che negli ultimi dieci anni la conoscenza dell' italiano tra gli studenti sloveni è diminuita notevolmente. Affermazioni simili si possono fare analizzando anche la seconda e la terza colonna della tabella che indicano la percentuale dei candidati 
Tabella ı: Risultati dell'esame di maturità di lingua italiana a livello avanzato tra gli anni 1995 e 2018 (Fonte: RIC 2019).

\begin{tabular}{|c|c|c|c|c|c|}
\hline Anno & 8 punti $(\%)$ & 6 punti (\%) & Soglia voto positivo (\%) & N. candidati & Voto medio \\
\hline 1995 & 22,61 & 40,87 & Dati non disponibili & II5 & 3,76 \\
\hline 1996 & 15,23 & 25,14 & Dati non disponibili & 155 & 3,12 \\
\hline 1997 & 9,64 & 30,12 & Dati non disponibili & 85 & 3,22 \\
\hline 1998 & 13,00 & 37,00 & Dati non disponibili & 100 & $3,5 \mathrm{I}$ \\
\hline 1999 & 13,98 & 35,48 & 60 & 93 & 3,52 \\
\hline 2000 & 30,59 & 45,88 & 55 & 87 & 4,05 \\
\hline 2001 & 20,45 & 42,05 & 55 & 88 & 3,75 \\
\hline 2002 & 8,60 & 57,30 & 55 & 113 & 3,68 \\
\hline 2003 & 14,4 & 54,20 & 55 & 122 & 3,75 \\
\hline 2004 & 5,40 & 51,60 & 57 & 109 & 3,64 \\
\hline 2005 & 14,10 & 58,60 & 54 & 66 & 3,82 \\
\hline 2006 & 22,50 & 40,83 & 54 & II5 & $3,8 \mathrm{I}$ \\
\hline 2007 & 9,00 & 53,63 & 50 & 104 & 3,69 \\
\hline 2008 & 8,80 & $23,8 \circ$ & 50 & 107 & 4,19 \\
\hline 2009 & 6,25 & 23,75 & 50 & $7 \mathrm{I}$ & 3,92 \\
\hline 2010 & 6,38 & 15,90 & 50 & 80 & 3,73 \\
\hline 2011 & 4,50 & 24,00 & 50 & 66 & 3,77 \\
\hline 2012 & 8,10 & 22,40 & 50 & 54 & 3,69 \\
\hline 2013 & 6,98 & 25,58 & 50 & 43 & 4,02 \\
\hline 2014 & 7,84 & 25,49 & 50 & 46 & 3,83 \\
\hline 2015 & 5,26 & 21,05 & 50 & 35 & 3,62 \\
\hline 2016 & 16,67 & 33,33 & 50 & 30 & 4,05 \\
\hline 2017 & 25,00 & 35,00 & 50 & 20 & 4,28 \\
\hline 2018 & 5,26 & 36,84 & 50 & 19 & 3,64 \\
\hline
\end{tabular}

che all'esame hanno conseguito 8 o 6 punti. Nonostante la soglia minima per il voto positivo del $60 \%$, nel 1999 i risultati della maturità erano ancora molto buoni. Il $35,48 \%$ dei candidati ha conseguito 6 punti, mentre il $13,98 \%$ ha ottenuto il punteggio massimo. Similmente, anche i risultati del 2000 erano eccezionali, anche se la percentuale dei punti necessari per il voto positivo è calata al 55 . Il 45,88\% dei candidati ha conseguito 6 punti, il $30,59 \%$ ha conseguito 8 punti e il voto medio ottenuto dagli studenti si è fermato a 4,05. Dalla tabella si può dedurre che fino al 2008 i risultati dell'esame di maturità di lingua italiana a livello avanzato erano ancora soddisfa- centi, mentre in seguito la situazione è cambiata drasticamente. Tra gli anni 1995 e 2007 , in media, il $15,35 \%$ dei candidati ha raggiunto il punteggio massimo ( 8 punti) e il $44,05 \%$ ha conseguito 6 punti, mentre tra gli anni 2008 e 2018 solamente $9,19 \%$ dei candidati ha ottenuto 8 punti e la percentuale degli studenti con 6 punti è calata al 26,10\%. In più, dopo il 2006 la soglia minima per il raggiungimento del voto positivo è crollata del $4 \%$ fermandosi al $50 \%$.

Statisticamente, i risultati hanno cominciato a migliorare nel 2016 quando il $16,67 \%$ dei candidati ha ottenuto 8 punti e il $33,33 \%$ ha conseguito 6 punti. La situazione è migliorata ancora di 
più nell'anno seguente in cui il 25,00\% degli studenti ha addirittura raggiunto il punteggio massimo e $35,00 \%$ ha ottenuto 6 punti. Il voto medio del 2017 si è così fermato a 4,28 rappresentando fino ad oggi il voto medio più alto sin dall'introduzione dell'esame di maturità nel sistema scolastico in Slovenia. Purtroppo, questi risultati ottimistici non rispecchiano la situazione reale e non rappresentano un segno di miglioramento nella conoscenza della lingua italiana tra i giovani sloveni. Analizzando i rapporti annuali sul rendimento degli studenti all'esame di maturità in lingua italiana a livello avanzato, disponibili sul sito Internet del RIC, si possono, infatti, trarre conclusioni molto diverse da quelle offerte dai dati statistici. Proprio nel rapporto del 2016 (ma anche del 2014 e del 2015) i valutatori esterni osservano che negli ultimi anni il grado di difficoltà dell'esame di maturità di lingua italiana è stato ridotto notevolmente rispecchiando la sempre più scarsa padronanza della lingua italiana tra i candidati. Per questo non sorprende che il voto medio ottenuto all'esame tra gli anni 1995 e 2007 era 3,64, mentre tra gli anni 2008 e 2018 è salito a 3,89. Questi dati ottimistici possono essere quindi attribuiti alla riduzione della difficoltà degli esami e al sempre più basso numero degli studenti che scelgono di sostenere l'esame di maturità di lingua italiana. A questo punto è necessario esporre anche le constatazioni dei valutatori esterni nel rapporto del 2012 in cui essi rilevano che il miglioramento nel rendimento dei candidati è causato pure dalla riduzione di criteri di valutazione. In più, negli ultimi anni i valutatori osservano tra i candidati la forte influenza di altre lingue straniere, in particolare inglese e spagnolo, e una pessima conoscenza delle regole ortografiche, sintattiche e morfologiche la quale può essere attribuita a un'enfasi eccessiva sull'approccio comunicativo-funzionale all'insegnamento di una lingua straniera.

\section{Lo schiacciante predominio dell' inglese come prima lingua straniera}

Il fenomeno linguistico più evidente a cui si assiste oggi è il fenomeno di "minoritarizzazione" di tutte le lingue rispetto all'inglese. L'inglese è diventato la lingua veicolare internazionale che svolge il ruolo di lingua globale facilitando i contesti interculturali. Con la nascita dei computer, degli smartphone, dei tablet e della TV moderna, l'inglese è diventato anche la lingua della rivoluzione tecnologica e di Internet avvicinandosi così sempre di più ai giovani. La forte presenza, nella vita quotidiana, dell'inglese è sicuramente uno dei fattori principali che hanno causato un notevole declino nella conoscenza della lingua italiana. Fino a non molto tempo fa, i giovani dell'Istria slovena guardavano i cartoni animati, i film e altre trasmissioni di divertimento quasi esclusivamente in lingua italiana. Grazie a questo la maggior parte dei giovani sloveni entrava a scuola con una conoscenza dell' italiano piuttosto elevata o almeno con una base sufficientemente solida da permettere loro di sviluppare in seguito una vera padronanza della lingua. Con la nascita della TV moderna e l'introduzione dei canali specializzati in cartoni animati, film e serie TV in lingua inglese i bambini, invece, già dalla tenera età sono esposti prevalentemente all'inglese, mentre l'uso della lingua italiana è limitato quasi esclusivamente all'ambito scolastico. Un elemento importante per il predominio dell' inglese è rappresentato anche dai video giochi che oggi occupano la maggior parte delle giornate dei giovani e rappresentano sicuramente uno dei metodi più divertenti e interessanti per imparare questa lingua. In tal modo, i ragazzi sono esposti alla lingua quasi inconsapevolmente e sono immersi nel processo di apprendimento linguistico che si svolge in uno stato di tranquillità, divertimento e spensieratezza.

Come abbiamo già spiegato, nelle scuole slovene dell'area bilingue l'italiano viene insegnato come seconda lingua ovvero come lingua dell'ambiente sociale, perciò in teoria dovrebbe occupare un posto più importante nel curricolo scolastico rispetto all'inglese che è definito prima lingua straniera. Analizzando la tabella 2 si può dedurre velocemente che la situazione reale non coincide con le nostre aspettative. 
Tabella 2: Numero di lezioni annuali di inglese LS I e di italiano L2 nelle scuole dell'obbligo nel territorio bilingue (Fonte: Libro bianco su istruzione e formazione 20II).

\section{Classe Inglese LS I (n. lezioni) Italiano L L (n. lezioni)}

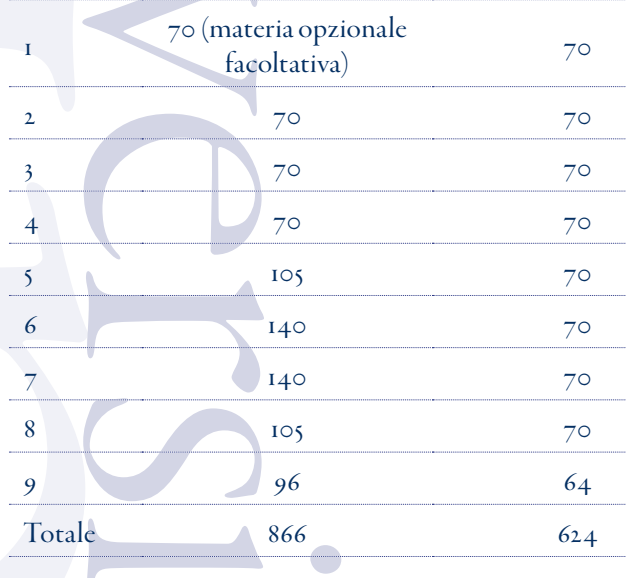

Gli alunni che frequentano le scuole elementari slovene dell'area bilingue seguono due volte alla settimana l'insegnamento dell'italiano come $\mathrm{L}_{2}$, il che corrisponde a 70 lezioni annuali. L'unica eccezione è rappresentata dalla nona classe in cui gli alunni hanno 6 lezioni di italiano in meno rispetto ad altre classi poiché per loro l'anno scolastico finisce prima. In totale, il curricolo prevede quindi 624 ore d'italiano $\mathrm{L}_{2}$ che sono distribuite uniformemente tra i nove anni scolastici. Dall'altra parte, i docenti d'inglese nelle scuole elementari slovene hanno a disposizione 866 ore per l'insegnamento d'inglese come LS I. Nella prima classe l'inglese è offerto come materia opzionale facoltativa, ma viene comunque scelto dalla maggioranza di alunni poiché l'insegnamento di questa lingua diventa obbligatorio nel secondo anno. Complessivamente, nel corso di nove anni scolastici gli alunni seguono 242 ore d'inglese $L S_{I}$ in più rispetto all'italiano L2. Non sorprende quindi che oggi l'attenzione da parte degli alunni e dai loro genitori è diretta quasi tutta all'inglese, dietro alla quale, ma a buona distanza, l'italiano e altre lingue.

\section{Modelli di rivitalizzazione di una lingua minoritaria}

Presentata la situazione preoccupante della lingua italiana nell'Istria slovena, in seguito vengono analizzati due modelli di rivitalizzazione che potrebbero offrire delle proposte per una strategia d'intervento a favore della lingua italiana nel territorio bilingue lungo la costa slovena. Si tratta del modello gallese e quello basco che hanno ottenuto negli ultimi anni il maggiore successo nell'ambito della pianificazione e dello sviluppo delle lingue minoritarie.A questo punto bisogna rilevare la consapevolezza che i risultati raggiunti con questa ricerca non si possono generalizzare completamente alle scuole slovene e che le strategie d'intervento usate in Galles e nei Paesi Baschi potrebbero non avere lo stesso successo nel sistema sloveno. Tuttavia, esse sicuramente offrono una panoramica di singoli esempi di buone pratiche nel campo del plurilinguismo, della multietnicità e della multiculturalità e potrebbero fungere come spunto per la rivitalizzazione della lingua italiana nell'Istria slovena.

\section{Il modello gallese}

Come osservano Baker e Prys Jones (2000, I23), per garantire la rivitalizzazione della lingua gallese, l'educazione della prima infanzia ha un ruolo vitale da svolgere. Il governo gallese assicura che tutti i bambini da 3 a 5 anni abbiano accesso gratuito all'educazione pre-scolastica potendo scegliere tra le scuole materne inglesi (il gallese viene insegnato come L2), gallesi o bilingui. Il governo dispone, inoltre, di un programma educativo rivolto a bambini tra $\mathrm{i} z \mathrm{e}$ i 7 anni nel quale lo sviluppo linguistico del gallese occupa la parte centrale. A questo punto bisogna porre l'accento sull'Associazione nazionale delle scuole dell'infanzia in Galles (Mudiad Ysgolion Meithrin), il principale fornitore prescolastico gallese che svolge un ruolo distintivo e importante sia nel rafforzare le capacità linguistiche dei bambini che parlano il gallese a casa sia nell'introdurre questa lingua tra i bambini non gallesi consentendo loro di seguire senza problemi le lezioni di gallese nelle scuole primarie. Nell'età presco- 
lare i bambini si trovano in un periodo linguisticamente delicato e flessibile in cui una lingua viene acquisita facilmente, naturalmente, spontaneamente e quasi inconsciamente. Per questo motivo, l'introduzione del gallese come $\mathrm{L}_{2}$ già nelle scuole materne è sicuramente un passo cruciale per la sua rivitalizzazione. I bambini, infatti, già dalla tenera età vengono così esposti al gallese acquisendolo giocando, cantando e guardando i cartoni animati. In questo modo, l'apprendimento della lingua non suscita sentimenti negativi e permette ai bambini di esprimersi senza paura di commettere errori. L'associazione Mudiad Ysgolion Meithrin è finanziata dal governo che partecipa attivamente allo sviluppo della lingua minoritaria offrendo persino corsi di formazione gratuiti per l'intero personale educativo. L'obiettivo di questi corsi è garantire al personale almeno un' istruzione di base in gallese, in modo che i bambini ricevano una buona introduzione della lingua già nell'età prescolare (Sterk, van der Meer e van Dongera 2017, IOI e Jones 200I, I4).

La promozione al bilinguismo e la consapevolezza dell' importanza della lingua gallese continua anche nelle scuole primarie. Nelle scuole con lingua d'insegnamento inglese il gallese viene insegnato come lingua seconda. A differenza delle scuole slovene del territorio bilingue in cui l'italiano non rappresenta altro che una materia obbligatoria prevista dal curricolo, in queste scuole il gallese occupa un posto molto più importante. L'apprendimento del gallese è, infatti, esteso su tutto il curricolo: i bambini, ad esempio, usano le tecnologie per l'informazione e la comunicazione (TIC) per sviluppare o migliorare la loro padronanza del gallese che viene spesso introdotto anche in matematica e altre materie del curricolo. Sebbene l'inglese sia la prima lingua in queste scuole, il gallese è frequentemente usato con l'obiettivo di migliorare le abilità linguistiche nelle interazioni quotidiane tra gli alunni. Alcune scuole assumono persino insegnanti gallesi la cui presenza può essere vista come una buona pratica in quanto garantisce agli alunni di ricevere un insegnamento di alta qualità. Con l'intenzione di promuovere la lingua gallese, negli ultimi trent'anni è stata sviluppata una vasta gamma di materiali didattici pubblicati in stampa, in digitale o in formati accessibili per gli alunni ciechi e ipovedenti. Nel 2012 è stata creata, inoltre, una piattaforma di apprendimento digitale nota come $H w b$ disponibile in tutto il Galles che offre un'ampia scelta di strumenti e risorse a livello nazionale, mentre nel 2014 il governo gallese ha avviato lo sviluppo di un'applicazione digitale per l'apprendimento della lingua gallese disegnata per i tablet e gli smartphone.A questo punto vale menzionare anche il National Language College, un'istituzione nazionale collegata con tutte le università del Galles che organizza corsi di lingua gallese, offre borse di studio, svolge delle ricerche scientifiche e garantisce dei materiali didattici tra i quali spicca una piattaforma di e-learning chiamata Y-Porth. Per quanto riguarda il materiale didattico per le scuole materne, la situazione è simile a quella delle scuole primarie. Esistono, infatti, molte varietà di materiali per l'insegnamento prescolare del gallese, inclusi i libri, i giocattoli, i DVD, i programmi televisivi e i video giochi. Le risorse didattiche di alta qualità sono comunque limitate (soprattutto rispetto a quelle inglesi) e il loro sviluppo rappresenta una sfida continua. Per questo motivo, molti insegnanti si assumono la responsabilità di produrre il proprio materiale in modi innovativi e attraenti (Van Dongera, Van derMeer e Sterk 2017, 102-104).

In seguito, gli stessi autori (2017, 104) rivelano un'altra particolarità del sistema educativo gallese riguardante gli alunni stranieri. Essi provengono da tutte le parti del mondo (dall'Europa, dall'Asia, dall'Africa, dal Nord e dal Sud America) integrandosi facilmente nell'ambiente gallese grazie a un sistema innovativo e accogliente. Il governo ha sviluppato, infatti, centri specializzati per gli alunni tra i 7 e gli i anni che si sono trasferiti in Galles tramite i quali vengono organizzati corsi intensivi a tempo pieno di immersione linguistica che durano quattro mesi. A fine corso gli alunni possono facilmente entrare sia nelle scuole con lingua d'insegnamen- 
to inglese sia in quelle con lingua d' insegnamento gallese.

\section{Il gallese alle famiglie}

Il sistema educativo rappresenta uno dei due pilastri più importanti nel processo della pianificazione linguistica: l'altro è la famiglia. Sarebbe, infatti, improbabile che il gallese (o qualsiasi altra lingua) potesse prosperare se la sua rivitalizzazione dipendesse solamente dalle scuole e non fosse sostenuta anche dalle famiglie e dall'ambiente sociale. Per qualsiasi lingua minoritaria per avere un futuro, i genitori devono riprodurre la lingua nei propri figli ed essa deve essere parlata e usata anche fuori dall'ambito scolastico.

La crescita, la prosperità e l'attuale stabilità del bilinguismo gallese sono dovute a un complesso insieme di fattori interagenti. Attraverso le proteste non violente svolte dagli attivisti linguistici nell'arco degli ultimi sessant'anni le autorità educative locali sono state incoraggiate e persino costrette a instaurare e promuovere le scuole bilingui. Comunque, dietro queste campagne c'è sempre stato un sostegno crescente da parte dei genitori. Come affermano Baker e Jones (2000, 120), molti di essi considerano l'educazione bilingue ovvero la flessibilità di parlare due lingue e condividere due culture come una fonte di migliori prospettive occupazionali e maggiori possibilità di benessere. Comunque, questa motivazione economica tra i genitori non fiorisce automaticamente ma viene sollecitata dalla situazione sul mercato di lavoro.

Il comitato nazionale di lingua gallese ha recentemente lanciato due progetti di marketing linguistico innovativi a livello internazionale per affrontare questa sfida. Si tratta del programma $T W F$, che significa "crescita" in lingua gallese, ma le lettere della parola in inglese stanno anche per "Taking Welsh to Families" (portando il gallese alle famiglie). Come spiegano Evas, Morris e Whitmarsh (2017, 37-38), nel Galles, attraverso questo progetto, i genitori vengono informati sui vantaggi connessi all'uso precoce di due lingue in famiglia e ricevono consigli su come crescere i figli in modo bilingue. Gli operatori del
TWF lavorano a stretto contatto con le cliniche ostetriche e gli enti di assistenza sanitaria fornendo sessioni di supporto per i genitori ed i loro bambini in cui viene posta enfasi sul valore del bilinguismo e sui benefici dell'educazione bilingue. Le donne incinte o quelle che hanno appena partorito usufruiscono di consigli sulle risorse utili a disposizione, come libri e CD, e viene offerto loro materiale informativo (ad esempio, il volantino promozionale Two Languages: Twice the Choice - Due lingue: doppia possibilità) a titolo gratuito. ${ }^{2}$ Il programma $T W F$ prevede anche la formazione professionale di ostetriche e assistenti domiciliari sull'importanza del bilinguismo che all' interno dell'ambiente domestico promuovono i vantaggi della conoscenza precoce di entrambi le lingue.

Secondo Baker e Jones (2000, I20), per ottenere risultati positivi, oltre al sistema educativo e al sostegno delle famiglie e dell'ambiente sociale, anche la politica deve contribuire alla pianificazione e alla rivitalizzazione di una lingua minoritaria. Negli ultimi sessant'anni, infatti, le istituzioni governative in Galles hanno guidato e incoraggiato la promozione del gallese come lingua minoritaria proponendosi l'obiettivo di raggiungere un milione di parlanti di gallese nel 2050. Proprio per questo motivo, il successo del bilinguismo in Galles può essere in parte attribuito al governo che, tramite leggi adeguate, finanziamenti e una serie di sistemi di supporto istituzionale, rende possibile la diffusione del gallese tra un numero di persone sempre più alto. Riguardo a questo, bisogna menzionare soprattutto il crescente ruolo degli ispettori per l'istruzione, dei consulenti delle autorità locali e dei presidi, aperti alle novità nonché la sempre più grande influenza delle istituzioni come, ad esempio, il Welsh Language Board, il Welsh Joint Education Commitee e lo Schools Council of Wales. Tali istituzioni, incoraggiate dal sostegno del governo, finanziano e producono materiali didattici in lingua gallese disponibili per ogni fascia di età. Da parte del governo gallese è sta-

I cosiddetti Bounty packs ovvero scatole e borse regalo contenenti campioni gratuiti di prodotti e informazioni sulla trasmissione intergenerazionale di gallese per le future mamme. 
to inoltre istituito un fondo di sviluppo (Development Fund) che assicura i finanziamenti per la realizzazione dei progetti rivolti allo sviluppo del bilinguismo.

\section{Il modello basco}

Il successo del modello gallese nel processo della pianificazione e della rivitalizzazione di una lingua minoritaria ha ispirato anche il movimento Ikastolak (Itziar 2008) nei Paesi Baschi che sarà analizzato di seguito.

\section{Sistema educativo}

Similarmente al sistema educativo gallese, nella comunità autonoma basca operano tre modelli di bilinguismo: il modello A, in cui lo spagnolo è la lingua d'insegnamento mentre il basco rappresenta una materia obbligatoria curricolare alla quale vengono concesse da 3 a 5 ore settimanali; il modello $B$, in cui entrambi le lingue sono usate come lingue d'insegnamento e vengono trattate parimenti dal curricolo scolastico; e il modello $\mathrm{D}$ dove il basco è la lingua d'insegnamento mentre lo spagnolo rappresenta una materia obbligatoria curricolare alla quale vengono destinate da 4 a 5 ore settimanali (alcune scuole offrono tutti e tre i modelli dando ai genitori la possibilità di scegliere il modello più appropriato per i loro figli). ${ }^{3}$ Questo sistema consente agli alunni di raggiungere una conoscenza della lingua basca piuttosto elevata indipendentemente dal modello scelto. I tre modelli sono disponibili a tutti i livelli dell' istruzione obbligatoria cominciando già a livello prescolare per i bambini di età compresa tra i 3 e i 6 anni. L'istruzione di secondo livello inizia all'età di $\mathrm{I} 2$ anni e il periodo obbligatorio termina all'età di 16 anni seguito poi da un ulteriore periodo non obbligatorio di 2 anni. All'inizio, il modello D è stato ideato primariamente per i madrilingue baschi, ma

Gli studiosi stranieri che si occupano del sistema educativo basco si chiedono spesso perché non esiste un modello C. La risposta più frequente è che l'alfabeto basco non contiene la lettera C. In realtà, all' inizio esisteva anche il modello $\mathrm{C}$, ma è stato rapidamente abbandonato per motivi organizzativi. L'alfabeto basco contiene la lettera $\mathrm{C}$, sebbene il suo uso sia limitato solamente alle parole straniere. la composizione degli alunni è cambiata notevolmente da allora dato che il numero di ragazzi con basco come L2 che scelgono questo modello sta aumentando rapidamente. Secondo i genitori che condividono il parere degli esperti, il modello D offre agli alunni migliori opportunità di padroneggiare entrambe le lingue (Ní Chuaig et al. 2015, 25-26).

Le ricerche sull'efficacia dei modelli svolte da Lasagabaster (200r) e Darquennes (et al. 2013) mostrano che il modello $\mathrm{D}$ è il più efficace per l'apprendimento del basco. Gli studenti del modello D sono, infatti, quelli che raggiungono punteggi più alti in lingua basca e sono quindi più vicini al bilinguismo equilibrato con alto livello di competenza in entrambe le lingue. L'ottima conoscenza del basco da parte di questi alunni non sorprende tanto dato che il modello D prevede il basco come lingua d'insegnamento. Sono però molto più interessanti le constatazioni fatte da Gardner (2000) secondo cui non esistono alcuni indizi mostranti che nel modello $\mathrm{D}$ lo sviluppo dello spagnolo sia ostacolato. Al contrario, non è stata osservata alcuna differenza tra le competenze linguistiche degli studenti in spagnolo nei tre diversi modelli.

\section{Il modello dei tre partner}

Come rileva Coyos $(2008,2-3)$ e come è già stato constatato dal modello gallese, il processo della pianificazione e della rivitalizzazione di una lingua minoritaria richiede un'interazione e una collaborazione incessante di tre protagonisti che tengono in mano le redini. In altre parole, il governo, insieme alle autorità regionali e locali, è responsabile di adottare le misure necessarie, di prendere delle decisioni giuste e di assicurare il sostegno finanziario, il sistema educativo è responsabile di realizzare le decisioni fatte dalle autorità, mentre i cittadini, cioè la società, rappresentano i destinatari ovvero i beneficiari del processo e sono sicuramente il partner più difficile da mobilitare. Per ottenere risultati positivi e soddisfacenti è necessario che questi protagonisti interagiscano tutti insieme. 
Il governo della comunità autonoma basca comprende diversi dipartimenti, ma per quanto riguarda la politica linguistica devono esserne menzionati almeno due: il Segretariato generale per la politica linguistica e il Dipartimento dell'educazione. Il primo è responsabile della supervisione della pianificazione linguistica e del finanziamento e del monitoraggio delle attività della CAB,${ }^{4}$ mentre il secondo si focalizza su diversi programmi per la rivitalizzazione della lingua basca, come ad esempio IRALE, GARATU, EIMA, NOLEGA e ULIBARRI.

Il programma IRALE è rivolto alla formazione professionale dei docenti in lingua basca, mentre il programma GARATU offre aiuto agli insegnanti sugli argomenti che devono essere affrontati in classe. Il programma EIMA è stato istituito dal governo nel 1982 per fornire assistenza finanziaria per promuovere la creazione di materiali didattici. Grazie ai finanziamenti sono stati sviluppati libri di testo, materiale audiovisivo e software e sono stati formati gruppi di lavoro composti da insegnanti e altri esperti che assicurano una vasta gamma di risorse didattiche per tutte le materie scolastiche. Per incoraggiare la partecipazione nei gruppi, i premi vengono assegnati a coloro che producono materiali di alta qualità. La qualità del lavoro svolto viene valutata dal governo prima del pagamento finale. $\mathrm{Nel}_{2004}$, ad esempio, sono stati erogati finanziamenti per 269 libri (o altri supporti di stampa), 30 video, Io audiocassette, 5 programmi software per computer, I8 CD-ROM multimediali e is premi per materiali sviluppati online. Essendo consapevoli del fatto che i materiali didattici per le lingue minoritarie come il basco hanno un mercato molto limitato rispetto alle lingue maggioritarie, i passi fatti dalle autorità basche risultano ancora più importanti. Il programma NOLEGA è stato istituito nel 1984 per assistere alla promozione del basco nelle scuole. Il programma offre sovvenzioni per sostenere attività legate alla cultura basca (teatro, canto, film, letteratura, tradizioni ecc.), stabilisce legami con organizzazioni private in modo che gli studenti possano studiare film baschi, leggere testi baschi e incontrare autori famosi ed offre agli alunni attività extracurricolari compresi gli scambi tra scuole di diverse aree sociolinguistiche. Il programma ULIBARRI (o HNP), che riunisce rappresentanti di ogni livello del sistema educativo, è iniziato nell'anno accademico 1996 I997 e mira alla rivitalizzazione della lingua basca nelle scuole attraverso lo sviluppo di attività specifiche. Ogni scuola deve disporre, infatti, del proprio progetto rivolto alla normalizzazione della lingua basca in cui partecipano anche i genitori e gli alunni, mentre il compito dell'ULI$B A R R I$ è di riunire i progetti di tutte le scuole in un unico piano linguistico sotto l'egida del sistema educativo. Il piano consiste di obiettivi realistici per un anno scolastico e di strategie su come raggiungerli. Alla fine dell'anno, tutte le scuole devono compilare dei questionari in relazione ad ogni obiettivo, mentre le autorità locali eseguono la valutazione del programma e forniscono alle scuole un feedback utile per l'anno scolastico successivo. Senza riguardo al modello (A, $\mathrm{B}$ o D) adottato dalla singola scuola, il programma ULIBARRI, che attualmente coinvolge 305 scuole con un totale di 128.763 alunni, invita, ad esempio, tutte le scuole a cambiare i software di tutti i computer in modo che essi siano nella lingua basca e di sistemare segni bilingui per i servizi igienici, per gli scuolabus, per le uscite ecc. Gli insegnanti sono invitati, inoltre, a introdurre nel loro curricolo l'insegnamento della cultura basca e di preferire il basco nelle attività extrascolastiche (sport, musica ecc.), mentre i genitori sono incoraggiati a prendere parte alle lezioni di lingua basca, a usare il basco a casa, a motivare i giovani a partecipare alle attività che promuovono la lingua minoritaria e a lasciare i propri figli guardare più spesso i programmi televisivi baschi (Aldekoa e Gardner 2002, 345-348).

Similmente al modello gallese, anche le scuole nei Paesi Baschi restano in stretto contatto con le famiglie e forniscono loro del materiale informativo su come crescere i propri figli in modo bilingue e promuovere la lingua e la cultura basca nella società. I responsabili sono consapevo- 
li che l'input in lingua basca offerto nelle scuole è molto importante, ma sarebbe ingenuo pensare che esso sia sufficiente a creare un ambiente bilingue. La situazione basca assomiglia molto al contesto in cui si trovano i giovani dell' Istria slovena. Similmente ai loro coetanei sloveni, anche molti giovani nei Paesi Baschi oltre all'ambiente scolastico non hanno altri contatti con la lingua minoritaria. Per questo motivo, ogni anno le autorità lanciano dei progetti ufficiali o meno, rivolti alla moltiplicazione delle possibilità in cui gli alunni vengono in contatto con la lingua basca fuori dall'ambiente scolastico. Si tratta di progetti che spesso coinvolgono anche l'Associazione dei Genitori e vengono promossi dalle organizzazioni, come ad esempio UEMA (consorzio per il supporto reciproco e l'assistenza legale), $A E K$ (istituto per la rivitalizzazione e l'insegnamento del basco agli adulti) e $H A B E$ (istituto per l'insegnamento del basco agli adulti). Tramite queste iniziative si rafforzano e migliorano i legami tra genitori, insegnanti e associazioni locali (Aldekoa e Gardner 2002, 349).

\section{Proposte per una strategia d'intervento a favore della lingua italiana}

Viste le premesse e le considerazioni esposte nei capitoli precedenti, in seguito vengono rilevate alcune proposte concrete per una strategia d'intervento a favore della lingua italiana nel territorio bilingue dell' 'Istria slovena.

Per risvegliare l'amore, l'interesse e l'atteggiamento positivo verso la lingua italiana tra la popolazione dell'Istria slovena è opportuno ricorrere al modello dei tre partner ovvero prendere dei provvedimenti nel settore governativo, scolastico e sociale. A livello educativo, legato alla formazione delle giovani generazioni, sarebbe necessario introdurre contenuti d'insegnamento dell'italiano già in età precoce in modo da favorire l'acquisizione successiva della lingua italiana. L'introduzione dell'italiano come L2 già nelle scuole dell'infanzia sarebbe sicuramente un passo cruciale per la sua rivitalizzazione. A questo scopo il governo dovrebbe offrire corsi di formazione gratuiti per l'intero personale edu- cativo e fondare un'associazione simile al $M u$ diad Ysgolion Meithrin in Galles che avrebbe la possibilità di sviluppare iniziative e campagne di marketing per promuovere e mostrare i vantaggi dell'acquisizione dell'italiano in età prescolare. In questo modo, le scuole dell' infanzia avrebbero la possibilità di offrire un'immersione linguistica per accelerare l'apprendimento dell' italiano come $\mathrm{L}_{2}$ tra i bambini senza una conoscenza pregressa e di mantenere un elevato livello di padronanza linguistica tra i bambini che vengono in contatto con l'italiano a casa.

Nel settore dell'educazione primaria l'italiano non dovrebbe rappresentare solamente una materia obbligatoria prevista dal curricolo, ma dovrebbe, come lingua dell'ambiente sociale, occupare un posto molto più importante. Seguendo il modello gallese e basco sarebbe opportuno destinare più ore all'insegnamento della lingua italiana introducendola gradualmente anche in altre materie scolastiche e avvicinandola così all'inglese a cui vengono, al momento, destinate 242 ore in più. Comparando la situazione della lingua inglese nelle scuole dell'obbligo nel territorio bilingue con quella dell' italiano si può dedurre, inoltre, che anche gli insegnanti di lingua italiana dovrebbero avere la possibilità di organizzare le cosiddette classi a livello ovvero di formare gruppi omogenei meno numerosi. In questo modo, l'insegnamento diventerebbe sicuramente più facile ed efficace, permettendo agli insegnanti di seguire più attentamente le necessità individuali degli alunni. L'intero personale educativo potrebbe incoraggiare gli alunni a usare l'italiano anche durante gli intervalli e nel loro tempo libero offrendo degli adesivi o altri premi simbolici. Similmente ai Paesi Baschi, anche le scuole dell'Istria slovena potrebbero promuovere l'italiano nelle attività extrascolastiche (sport, musica ecc.) cambiando i software dei computer in modo che essi siano in lingua italiana e sistemando segni bilingui per i servizi igienici, per gli scuolabus, per le uscite ecc. Per offrire agli alunni un insegnamento di alta qualità le scuole potrebbero coinvolgere persone di madrelingua italiana che lavorassero accanto agli insegnanti come 
mediatori linguistici e culturali. Essi valorizzerebbero ancora di più i contenuti che promuovono i valori della convivenza interetnica e rappresenterebbero sicuramente un valore aggiunto. Con l'intenzione di migliorare la posizione della lingua italiana nel sistema educativo le scuole dovrebbero promuovere, con l'aiuto finanziario del governo, la creazione di materiali didattici. Sarebbe necessario lanciare un programma simile all'EIMA nei Paesi Baschi per sviluppare libri di testo, giochi e materiale multimediale e creare delle applicazioni (simili al Hwbin Galles) e dei software per i computer, i tablet e gli smartphone destinati all'apprendimento digitale della lingua italiana. Gli insegnanti e altri esperti potrebbero formare gruppi di lavoro che assicurerebbero una vasta gamma di risorse didattiche. Per incoraggiare la partecipazione, le autorità potrebbero assegnare dei premi a coloro che produrrebbero materiali di alta qualità. Per quanto riguarda gli individui provenienti da paesi esteri o da altre parti della Slovenia che non appartengono al territorio bilingue, le scuole potrebbero seguire il modello gallese e organizzare corsi intensivi d'immersione linguistica, mentre gli alunni con scarsa o senza conoscenza pregressa della lingua italiana dovrebbero essere aiutati con interventi di sostegno aggiuntivi.

Come suggeriscono anche Burra e Debeljuh $(2013,167)$, le direzioni delle scuole elementari e medie dell'Istria slovena dovrebbero incentivare e promuovere ancora di più gli incontri tra italofoni e slavofoni, l'accoglimento delle diversità e la reciproca conoscenza, in modo da eliminare le barriere che condizionano la libera manifestazione linguistica sul territorio. A questo scopo sarebbe sicuramente importante realizzare scambi tra scuole di diverse aree sociolinguistiche e organizzare colonie estive e soggiorni prolungati attraverso i quali gli alunni sperimentassero un'immersione linguistica in contesti sociali dove la lingua italiana è usata in modo naturale nella quotidianità. Per realizzare queste iniziative, già sperimentate nell'area anglosassone, sarebbe necessario senza dubbio il coinvolgimento attivo sia delle organizzazioni locali maggiori- tarie sia quelle minoritarie. Sarebbe importante incoraggiare, inoltre, la creazione di una cultura giovanile e di una rete d'uso informale della lingua italiana basato sul modello d'insegnamento extrascolastico del basco ULIBARRI. Le scuole dovrebbero sostenere anche attività legate alla cultura italiana (teatro, canto, film, letteratura, tradizioni ecc.), accrescere la consapevolezza del patrimonio linguistico degli italofoni e stabilire legami con organizzazioni private e pubbliche. In seguito, soprattutto nelle scuole medie, bisognerebbe organizzare incontri tra studenti, genitori e datori di lavoro accentuando che le competenze bilingui sono sempre più cercate, gradite e desiderate sia nel settore pubblico sia in quello privato e che la flessibilità di parlare più lingue offre migliori prospettive occupazionali e maggiori possibilità di benessere.

Nell'ambito sociale bisognerebbe realizzare un intervento precoce mirato al mantenimento del ricco patrimonio linguistico dell'Istria slovena. A tal fine, dovrebbero essere promossi programmi e progetti di sensibilizzazione linguistica rivolti a tutta la popolazione. Similmente al modello gallese (Taking Welsh to Families), la maggior enfasi dovrebbe essere posta sulle famiglie del territorio bilingue che dovrebbero essere motivate a trasmettere ai figli la lingua e la cultura italiana. A questo scopo le autorità dovrebbero informare i genitori sui vantaggi connessi all'uso precoce di due lingue e fornire consigli su come crescere i figli in modo bilingue. Le associazioni linguistiche dovrebbero lavorare a stretto contatto con le cliniche ostetriche e gli enti di assistenza sanitaria distribuendo materiali informativi e servizi di consulenza a domicilio, sia nella fase prenatale sia nei primi anni di vita del bambino. Il programma dovrebbe coinvolgere anche i genitori che non sanno parlare l'italiano e incoraggiarli a spingere i propri figli a guardare i programmi televisivi italiani e a partecipare alle attività extrascolastiche collegate con la lingua minoritaria. Le scuole potrebbero consigliare a questi genitori, inoltre, come aiutare i propri figli nel processo dell'apprendimento della lin- 
gua italiana ed invitarli a frequentare corsi di lingua italiana per adulti.

Siccome per ottenere risultati positivi e soddisfacenti nella rivitalizzazione e nella promozione di una lingua minoritaria è necessario coltivare un'interazione reciproca tra la sfera scolastica sociale e governativa, le proposte fatte sopra non possono essere realizzate senza l'aiuto del governo e delle autorità locali e regionali. Come suggeriscono anche Burra e Debeljuh (2013, 170), la legislazione connessa allo status della lingua italiana, intesa come lingua dell'ambiente, dovrebbe essere migliorata e implementata. Essa dovrebbe essere inquadrata all'interno della massima decentralizzazione del territorio nazionale, attribuendo alle autonomie locali competenze speciali nel settore amministrativo, educativo ed economico, attraverso la devoluzione dei poteri dal livello nazionale a quello regionale. A tale scopo sarebbe opportuno costituire una Regione a Statuto speciale, comprendente i comuni di Capodistria, Ancarano, Isola e Pirano, che assomiglierebbe al sistema basco e gallese. Per la sua realizzazione sarebbe sicuramente necessario anche il coinvolgimento attivo della comunità italiana residente sul territorio. A livello legislativo bisognerebbe, inoltre, emanare una legge sull'uso della lingua italiana, che prenderebbe spunto dal disegno di legge gallese $\mathrm{Wel}$ sh language act. Questa legge conferirebbe ad un nuovo organismo, istituito come ente pubblico non ministeriale su modello del Welsh language board, le competenze esposte nei vari settori d'intervento.

La Regione a Statuto speciale menzionata sopra dovrebbe collaborare strettamente sia con il governo sloveno sia con quello italiano. Tutte e tre le parti dovrebbero contribuire alla pianificazione e alla rivitalizzazione della lingua italiana nell'Istria slovena fornendo fondi per sostenere le iniziative linguistiche e per produrre materiali didattici in lingua italiana di alta qualità. A tale fine sarebbe bene imitare la mossa del governo gallese e istituire un Development Fund che potrebbe assicurare i finanziamenti per la realiz- zazione dei progetti rivolti allo sviluppo del bilinguismo.

\section{Conclusione}

L'analisi dei due esempi di buona pratica che hanno permesso di ottenere i migliori risultati nell'ambito della rivitalizzazione delle lingue minoritarie in Galles e nei Paesi Baschi suscita speranza che, allo stato attuale, esistano ancora dei margini per un intervento efficace anche per la lingua italiana nell'Istria slovena. A questo scopo è fondamentale agire partendo da una pianificazione programmata e relativa concretizzazione ben strutturata nei vari settori d'intervento. In tal senso è di grande importanza una presa di coscienza e responsabilizzazione sia della popolazione italofona sia di quella maggioritaria. Benché i provvedimenti adottati nell'ambito scolastico, insieme allo sforzo, alle iniziative e alla premura degli insegnati del territorio bilingue rappresentino sicuramente un pilastro molto importante nella rivitalizzazione della lingua italiana nell'Istria slovena, non possiamo aspettarci una soluzione che provenga solamente dal sistema educativo. Esistono, infatti, due assi: un asse dinamico comprendente gli studenti e i loro genitori che dopo un certo numero di anni lasciano la scuola e un asse stabile (l'amministrazione, gli insegnanti, i cittadini ecc.) che resta sul campo più a lungo. Tutti gli interventi effettuati sull'asse dinamico sono in un certo senso temporanei, mentre quelli effettuati sull'asse stabile sono sicuramente più efficaci e hanno una durata molto più lunga. I risultati della nostra ricerca hanno mostrato che le modalità e i contenuti di un intervento di successo dovrebbero sorgere dal modello dei tre partner secondo il quale è necessario prendere dei provvedimenti nel settore governativo, scolastico e sociale. Il sostegno istituzionale fornito dallo stato o dal governo locale è molto importante, se non di vitale importanza. Le autorità hanno almeno tre ruoli principali: prendere decisioni, finanziare progetti e adottare - o meno - leggi linguistiche a favore della lingua minoritaria dando legittimità al progetto di rivitalizzazione. Tuttavia, anche la politica 
istituzionale non può ottenere risultati positivi senza la partecipazione attiva della comunità ovvero senza il coinvolgimento e l'impegno dei cittadini. Essi rendono viva la lingua usandola nella vita quotidiana stimolando così automaticamente il processo di rivitalizzazione. Per ottenere risultati soddisfacenti e migliorare la situazione dell' italiano come lingua seconda nell'Istria slovena è quindi necessario sviluppare e promuovere un'interazione e una collaborazione incessante tra questi tre protagonisti.

\section{Povzetek}

Glavni namen članka je bil predstaviti bralcu opis zaskrbljujočega položaja italijanščine kot drugega jezika vzdolž slovenske obale ter ponuditi konkretne rešitve za revitalizacijo in izboljšanje italijanščine med mladimi na dvojezičnem območju. Glede na število ur, ki so v šolah slovenske Istre namenjene učenju italijanščine, ter bivanje v dvojezičnem okolju, kjer se ljudje redno srečujejo z italijanskim jezikom, bi morali dijaki slovenske Istre po trinajstih letih šolanja doseči visoko stopnjo znanja jezika, v resnici pa je stanje zaradi različnih dejavnikov ravno obratno. Eden glavnih ciljev raziskave je bil poiskati rešitve in predstaviti konkretne predloge za revitalizacijo in izboljšanje položaja italijanščine, zaradi česar je bila opravljena natančna analiza baskovskega in valižanskega modela, ki sta na področju večjezičnosti in promoviranja manjšinskih jezikov na politični, šolski in družbeni ravni v zadnjih letih doživela največji uspeh. Rezultati analize so pokazali, da je mogoče ljubezen, zanimanje in pozitiven odnos do manjšinskega jezika prebuditi $s$ pomočjo modela treh partnerjev oziroma z uvajanjem ustreznih ukrepov na področju politike, šolstva in družbe. Na področju izobraževanja bi tako bilo treba poskrbeti za zgodnje uvajanje italijanskega jezika v vrtce in prostočasne dejavnosti, spodbujati razvoj modernejših didaktičnih pripomočkov, organizirati intenzivne jezikovne tečaje, nuditi individualno pomoč ter spodbujati druženje slovenskih in italijanskih otrok. Na družbenem področju bi morala država spodbujati programe in projekte za ozaveščanje pomembnosti znanja jezikov, največ pozornosti pa bi bilo treba po baskovskem in valižanskem modelu nameniti mladim družinam. Jezikovne institucije bi morale tesno sodelovati s porodnišnicami, kjer bi bodočim staršem s pomočjo informativnih letakov in osebnega svetovanja predstavili pomen dvojezičnostiv slovenski Istri ter jih tako spodbudili, da bi svoje otroke vzgajali v duhu jezikovne in kulturne raznolikosti. Uresničevanje večine zgoraj omenjenih predlogov je seveda v veliki meri odvisno od državne in lokalne politike, ki bi morali poskrbeti zlasti za zakonsko in finančno podporo. Država bi morala večjo pristojnost dodeliti regionalnim oziroma lokalnim institucijam vzdolž slovenske obale, ki bi bile združene v enotno regijo s posebnim statutom. V realizacijo nove zakonodajne podlage, ki bi ščitila status italijanskega jezika in zagotavljala njegovo praktično uporabo v tem prostoru, bi bilo treba aktivno vključiti koprsko, izolsko, piransko in ankaransko občino ter italijansko skupnost.

\section{Summary}

The main purpose of the article was to acquaint the reader with the alarming position of Italian as a second language along the Slovenian coast and to offer concrete solutions for the revitalization and improvement of Italian among young people in the bilingual area. $\mathrm{Ta}$ king into account the number of lessons of Italian in the schools of Slovene Istria and the fact that this area is considered a bilingual environment where people regularly speak Italian, after thirteen years of schooling Slovenian students should achieve a high level of language skills. However, due to various reasons, the situation is completely opposite. Since one of the main purposes of the paper was to offer concrete solutions for the revitalization and improvement of Italian as a second language, two examples of good practice - the Welsh and Basque model - in the field of minority language revitalization were analyzed. The success of these two models can fill us with hope that in the current situation, there is still room for an effective intervention for the Italian language. The results of the analysis showed that love, interest and a positive attitude towards a minority language could be restored by the three partners model or by introducing appropriate measures in the field of politics, education and society. As far as the educational system is of our concern, the early introduction of the Italian language in kindergartens should be ensured, the development of modern didactic tools should be encouraged, individual pupil assistance should be provided and encounters between Slovene and Italian children should be promoted. In the social environment, the government 
should promote programs and projects whose aim is to emphasize the importance of language skills focusing mainly on young families. Language institutions should cooperate closely with maternity hospitals offering prospective parents informative leaflets and personal counseling about the importance of bilingualism in Slovene Istria and encouraging them to raise their children in a spirit of linguistic and cultural diversity. The implementation of the abovementioned proposals is largely dependent on national and local policies which should provide legal and financial support. The government should distribute greater authority to regional and local institutions along the Slovenian coast and establish a single region with a special statute. The Koper, Izola, Piran and Anakran municipalities and the Italian community should be actively involved in the implementation of the new legislative basis, which would protect the status of the Italian language and ensure its practical use in this area.

\section{Riferimenti bibliografici}

Aldekoa, J., e N. Gardner. 2002. “Turning Knowledge of Basque into use: Normalisation Plans for Schools." International Journal of Bilingual Education and Bilingualism, 5 (6): 339-354.

Baker, C., e P. J., Meirion. 2000. "Welsh Language Education: A Strategy for Revitalization." In Language Revitalization. Policy and Planning in Wales, a cura di Collin H. Williams, in6137. Cardiff: University of Wales Press.

Balboni, P. E. 1994. Didattica dell'italiano a stranieri. Roma: Bonacci editore.

Balboni, P. E. 2008. Le sfide di babele. Insegnare le lingue nelle società complesse. Torino: UTET Università.

Balboni, P. E. 2014. Didattica dell'italiano come lingua seconda e straniera. Torino: Loescher Editore.

Burra, A., e A. Debeljuh. 2013. L'italiano nelle aree di confine. Analisi e proposte per la sua rivitalizzazione. Capodistria: Centro Italiano di Promozione, Cultura, Formazione e Sviluppo “Carlo Combi”. Centro nazionale per gli esami RIC /
Državni izpitni center RIC. https://www. ric.si/.

Coyos, J. B. 2008. "Revitalization of the Basque language: the case of the Northern Basque Country. How to reinforce synergy between agents of the linguistics policy, authorities and society?" Language Emancipation of Historical Minorities Nordic-French workshop - Maison des Sciences de l'Homme, 2008 (IO): I-8.

Čok, L. 2009. "Italijanščina kot drugi jezik in sredstvo sožitja ob angleščini kot prvem tujem jeziku.” In Izobraževanje za dvojezičnost v kontekstu evropskih integracijskih procesov, a cura di Lucija Čok, 89-ro2. Koper: Annales.

Darquennes, J., I. Martinez de Luna, P. Suberbiola, e M. Zalbide. 2013. "The use of Basque in model D Schools in the Basque Autonomous Community." European Journal of Applied Linguistics, O: I-33. Gardner, N. 2000. Basque in Education in the Basque Autonomous Community. Bilbao: Eusko Jaurlaritzaren Argitalpen Zerbitzu Nagusia - Servicio Central de Publicaciones del Gobierno Vasco. Governo basco. 2019. Governo basco, Dipartimento di Educazione. https:// www.euskadi.eus/hasiera/.

Governo gallese. 2019. Governo gallese, Dipartimento di Educazione. https:// www.theschoolrun.com/welsh-teachingin-primary-schools.

Itziar, E. 2008. "Promoting the Minority Language Through Integrated Plurilingual Language Planning: The Case of the Ikastolas." Language Culture and Curriculum, August 2008: 85-IOI.

Jagodic, D. 2013. “L'apprendimento dello sloveno come lingua seconda o straniera in età adulta nella fascia confinaria del Friuli Venezia Giulia: indagine fra i partecipanti al corso." In Fra lingua seconda e lingua straniera. Insegnamento e apprendimento dello sloveno in età adulta nella fascia confinaria del Friuli Venezia Giulia, a cura 
di Devan Jagodic e Štefan Čok, 40-76.

Trieste: Associazione temporanea di scopo “jezik-lingua”.

Jones, M. P. 200I. Welsh: The Welsh Language

in Education in the UK. Ljouwert/

Leeuwarden: Mercator Education.

Kompara, M. 20r4. "Je slovenska Istra še dvojezična?" Jezikoslovni zapiski 2014 (20/2): 89-106.

Krek, J., e M. Metljak. a cura di 20 ir. Bela knjiga o vzgoji in izobraževanju v Republiki Sloveniji. Lubiana: Ministrstvo za šolstvo in šport. https://pefprints.pef.uni-lj. si/I195/I/bela_knjiga_2orr.pdf.

Lasagabaster, D. 200ı. "Bilingualism, Immersion Programmes and Language Learning in the Basque Country.".Journal of Multilingual and Multicultural Development, 22 (5): 40I-425.

Limon, D., e S. Novak-Lukanovič. 2012. "Language policy in Slovenia." Language Culture and Curriculum ,25(I): 27-39.

London: Linguistics Institute of Ireland, Taylor \& Francis (Routledge).

Luise, M. C. 2006. Italiano come lingua seconda. Elementi di didattica. Torino: UTET Università.

Miklič, T., e M. Ožbot. 200I. "L'insegnamento dell'italiano in Slovenia." Bulletin VALSASLA (Vereinigung für angewandte Linguistic in der Schweiz), 73: I13-I2I.

Neuchâtel: Centre de linguistique appliqué Université de Neuchâtel.

Ní Chuaig, N., L. Ní Thuairisg, C. Ó Brolcháin, e P. Ó Duibhir. 2015. Education Provision through Minority Languages: Review of International Research. Dublin: Department of Education and Skills.

Novak-Lukanovič, S. 1998. “Stališča do večinskega in manjšinskega jezika v vzgoji in izobraževanju na narodnostno mešanem območju v Sloveniji." In Jezik za danes in jutri: zbornik referatov na II. Kongresu, Ljubljana, 8.-10. Io. 1998, a cura di Inka Štrukelj, 9I-96. Ljubljana: Društvo za uporabno jezikoslovje Slovenije - Inštitut za narod nostna vprašanja.

Pišot - Čok, L. 1990. "Contributo alla didattica della lingua italiana come lingua Seconda.” Linguistica, 30 (I): 15I-167.

Sterk, R., C. van der Meer, e R. van Dongera. 2017. Research for CULT Committee Minority languages and education: best practices and pitfalls. Brussels: European Parliament, Policy Department for Structural and Cohesion Policies.

Ufficio statistiche della Repubblica di Slovenia SURS / Statistični urad Republike Slovenije. https://www.stat.si/statweb.

Zudič Antonič, N. 2007. "L'educazione letteraria in prospettiva interculturale: settore scientifico-disciplinare di afferenza: L-LIN/or linguistico-glottodidattico." Tesi di dottorato. Venezia: Università Ca' Foscari Venezia. 